\title{
Production of Neutron Rich Isotopes in Fission. A Study for RNB Facilities.
}

\author{
V.A. Rubchenya ${ }^{a}$, J. Äystö ${ }^{b}$ \\ ${ }^{a}$ Department of Physics, University of Jyväskylä, FIN-40351, Jyväskylä, Finland \\ ${ }^{\mathrm{b}}$ EP-ISOLDE, CERN, 1211 Geneva 23, Switzerland
}

\begin{abstract}
The theoretical model for calculations of the fission product yields in the light particle induced fission is discussed. A comparison of the production cross sections in fission of ${ }^{238} \mathrm{U}$ by protons, thermal and fast neutrons, and photons is presented. The results of studies of the very asymmetric fission of ${ }^{238} \mathrm{U}$ by protons and neutron at the moderate energy are reported.
\end{abstract}

\section{Introduction}

Nuclear fission is still an inexhaustible source for discovering, producing, and investigating exotic nuclei with high neutron excess $(1 ; 2)$. Fission of heavy nuclei is practically the only method for production of very neutron-rich nuclides in the atomic number range from $Z=27$ to $Z=65$. A few approaches for the production of neutron-rich nuclides are proposed in different Radioactive Nuclear Beam (RNB) projects. Among these the most important ones are:

- thermal-neutron induced fission of ${ }^{235} \mathrm{U}(3 ; 4)$;

- fast neutron induced fission of ${ }^{238} \mathrm{U}(5 ; 6)$;

- photon-induced fission of ${ }^{238} \mathrm{U}(7)$.

Comparative investigations of neutron-rich fission product yields in these different reactions are important for the development of RNB facilities. A reliable fission model is also important for the technical application of nuclear reactions at intermediate energy for the energy production and transmutation of nuclear waste in hybrid reactors (8).

During the last few years the systematic experimental and theoretical studies on charge and mass distributions of fragments in proton, deuteron, and neutron induced fission of ${ }^{238} \mathrm{U}$ at intermadiate energy have been carried out at the Accelerator Laboratory of the University of Jyvaskyla. A special attention has been paid to exploring extreme asymmetric fission where the light

Preprint submitted to Elsevier Preprint $\quad 8$ March 2001 
fragment mass $A \leq 80$. A new version of fission model for calculating fission product yields in the light particle induced fission reaction has been developed and tested. In this report the main results of this investigations will be presented. A model calculation analysis of fission product yields for the different RNB schemes will also be done especially for nulcides near the doubly magic nucleus ${ }^{78} \mathrm{Ni}$.

\section{Model}

The detailed theoretical calculation of the fission product formation cross sections at intermediate energy consists of two parts: (i) modelling the reaction mechanism to calculate mass, charge, and excitation energy distributions of compound nuclei and (ii) modelling the fission process itself. For the case of the light-particle induced fission of heavy nuclei the theoretical model for calculation of independent fission product cross sections was proposed and developed in our previous works $(9 ; 10 ; 11)$. The formation cross section of a fission product with mass number $\mathrm{A}$ and charge number $\mathrm{Z}$ can be expressed in the form

$$
\sigma_{f}(A, Z)=\sum_{A_{c} Z_{c}} \int Y_{i n d}(A, Z) d E_{c} \frac{d \sigma_{f}\left(A_{t}, Z_{t}, A_{p}, Z_{p}, E_{p}, A_{c}, Z_{c}, E_{c}\right)}{d E_{c}},
$$

where subscripts $t, p$ and $c$ refer to target, projectile and compound nuclei, respectively, $d \sigma_{f} / d E_{c}$ is the partial fission cross section of the compound nucleus at the excitation energy $E_{c}$ for different fission chances over which the summing is carried out and $Y_{\text {ind }}\left(A, Z, A_{c}, Z_{c}, E_{c}\right)$ is the independent yield. The independent yields $Y_{\text {ind }}$ are defined as the yields of fission products after light particle emission from excited primary fragments:

$$
\begin{array}{r}
Y_{\text {ind }}\left(A, Z, A_{c}, Z_{c}, E_{c}\right)=\sum_{a_{l c p}, z_{l c p}} P_{a_{l c p}, z_{l c p}}\left(A+a_{l c p}, Z+z_{l c p}, A_{c}, Z_{c}, E_{c}\right) \\
P_{p r e}\left(Z+z_{l c p}, A+a_{l c p}, A_{c}, Z_{c}, E_{c}\right) Y_{p r e}\left(A+a_{l c p}, A_{c}, Z_{c}, E_{c}\right) .
\end{array}
$$

Here $P_{a_{l c p}, z_{l c p}}\left(A+a_{l c p}, Z+z_{l c p}, A_{c}, Z_{c}, E_{c}\right)$ is a probability of emission of $\left(a_{l c p}, z_{l c p}\right)$ light particle from fragment with the mass $A+a_{l c p}$ and charge $Z+z_{l c p}$ numbers, $P_{\text {pre }}\left(Z+z_{l c p}, A+a_{l c p}, A_{c}, Z_{c}, E_{c}\right)$ is a charge distribution of the $A+a_{l c p}$ isobaric chain and $Y_{\text {pre }}\left(A+a_{l c p}, A_{c}, Z_{c}, E_{c}\right)$ is a primary fission fragment mass distribution. At the excitation energies $E_{c}$ up to about $150 \mathrm{MeV}$ the role of emission of proton and others particle is negligible in comparision with neutron emission. 
At low excitation energies, the primary fission fragment mass and charge distributions exhibit odd-even staggering. The primary distributions are presented in the factorised form

$$
P_{\text {pre }}(Z)=\tilde{P}_{\text {pre }}(Z) F_{\text {oe }}(Z), \quad Y_{\text {pre }}(A)=\tilde{Y}_{\text {pre }}(A) F_{\text {oe }}(A),
$$

where $\tilde{P}_{\text {pre }}(Z)$ and $\tilde{Y}_{\text {pre }}(A)$ are smoothed distributions, and the functions $F_{o e}(Z)$ and $F_{o e}(A)$ describe odd-even staggering. The method of modelling the smoothed mass distribution is based on the multimodal nature of nuclear fission (12). The four fission modes: symmetric, standard-I, standard-II and superasymmetric were taken in consideration to approximate the smoothed primary mass distribution:

$$
\tilde{Y}_{\text {pre }}(A)=C_{s} y_{s}(A)+C_{a 1} y_{a 1}(A)+C_{a 2} y_{a 2}(A)+C_{a 3} y_{a 3}(A)
$$

Here $y_{s}$ and $y_{a 1}, y_{a 2}, y_{a 3}$, are symmetric and asymmetric components which present contributions from different fission modes. Each asymmetric component consists of two Gaussians representing the heavy and light fragment mass groups. The component $y_{a 1}$ is connected with the magic number $Z=50$ and $N=82$ in the heavy fragments, and the superasymmetric component $y_{a 3}$ is influenced by the nuclear shells $Z=28$ and $N=50$ in the light fragments. The asymmetric mode $y_{a 2}$ is supposed to be connected with a "deformed" nuclear shell at $N=86-90$. The competition between fission modes is determined by fission dynamics and nuclear shells in the fission fragments. The parameters entered in eq. (4) had been approximated as functions of the excitation energy, mass and charge of the compound nucleus. The smoothed pre-neutron emission isobaric chain charge distribution is approximated by a Gaussian distribution, therefore odd-even structure can be described by the parameter defined as a third difference of the natural logarithms of the fractional yields (13).

The partial distribution of the compound nucleus ensemble in eq. (1) depends on the fast and pre-equilibrium stages of reaction and fission dynamics. The fission process had been treated by the method developed by us in (14) with inclusion of the influence of nuclear friction. In the calculations we have used the energy-dependent friction coefficient in the form proposed in (15). The excitation energy of the primary fission fragments was calculated in the famework of the scission point fission model. The model has been tested and used for the light particle induced fission at energies up to $100 \mathrm{MeV}$. Some results will be shown in the next sections. 


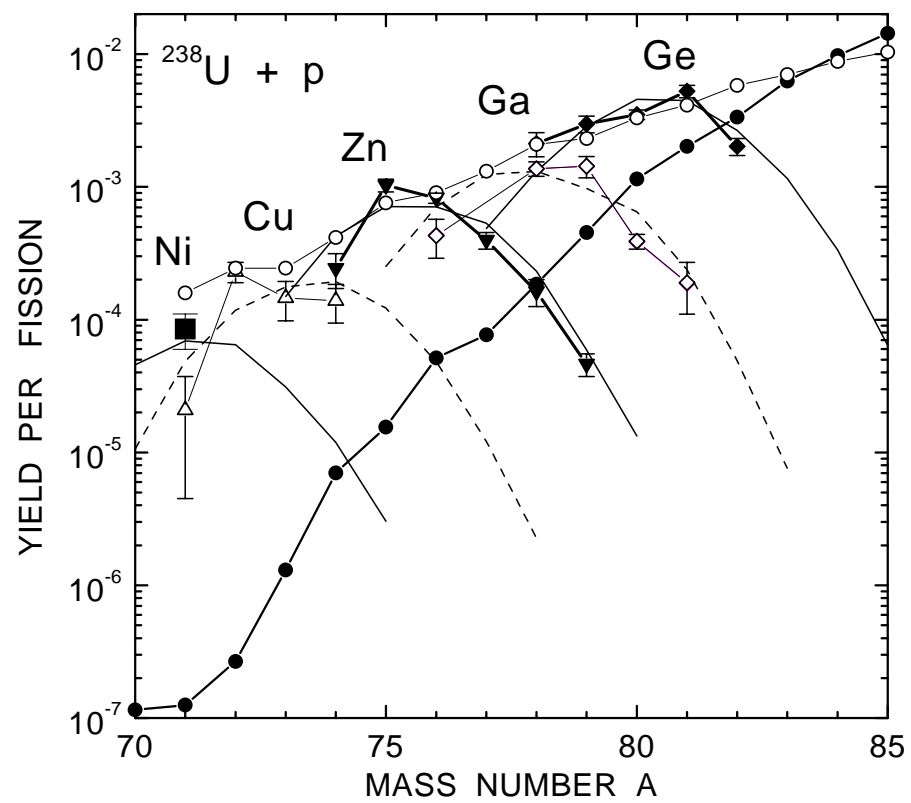

Fig. 1. Measured independent yields (points with error bars) of fission products in $25 \mathrm{MeV}$ proton induced fission of ${ }^{238} \mathrm{U}$, and the mass yields (solid circles) in the thermal neutron induced fission of ${ }^{235} \mathrm{U}$, and the pre-neutron emission mass distribution (open circles) at $E_{p}=35 \mathrm{MeV}$. The theoretical isotopic yields are shown by thin solid and dashed lines.

\section{Superasymmetric fission}

Information on extreme asymmetric fission of heavy nuclei, whith the light fragment mass $\mathrm{A}_{L}<80$, is scarce, mainly due to very low yields. Available information on the very asymmetric thermal neutron induced fission of heavy nuclei had recently been reviewed in (16). For the first time the independent yields of neutron-rich nuclei in the very asymmetric proton induced fission of ${ }^{238} \mathrm{U}$ at $E_{p}=25 \mathrm{MeV}$ had been measured using the ion guide isotope separation technique IGISOL (17). The mass distributions in the proton induced fission of ${ }^{238} \mathrm{U}$ at $E_{p}=20,35,50$ and $60 \mathrm{MeV}$ had also been measured by the time-of-flight method using the HENDES setup (11). The results of these experiments are presented in Fig. 1, where IGISOL data are shown by points with the errors bars and HENDES data for pre-neutron emission fragment distribution at $E_{p}=35 \mathrm{MeV}$ are shown by open circle. The theoretical results for independent yields are presented in Fig. 1 by lines in comparison with IGISOL data. One can see that the yields in the superasymmetric mass region for fission of Np compound nuclei at intermediate excitation energy differ drastically from the ones in low energy fission of ${ }^{236} \mathrm{U}$ compound nuclei. In a very asymmetric mass division at intermediate excitation energy $\left(\mathrm{E}_{c} \geq 20 \mathrm{MeV}\right)$ 


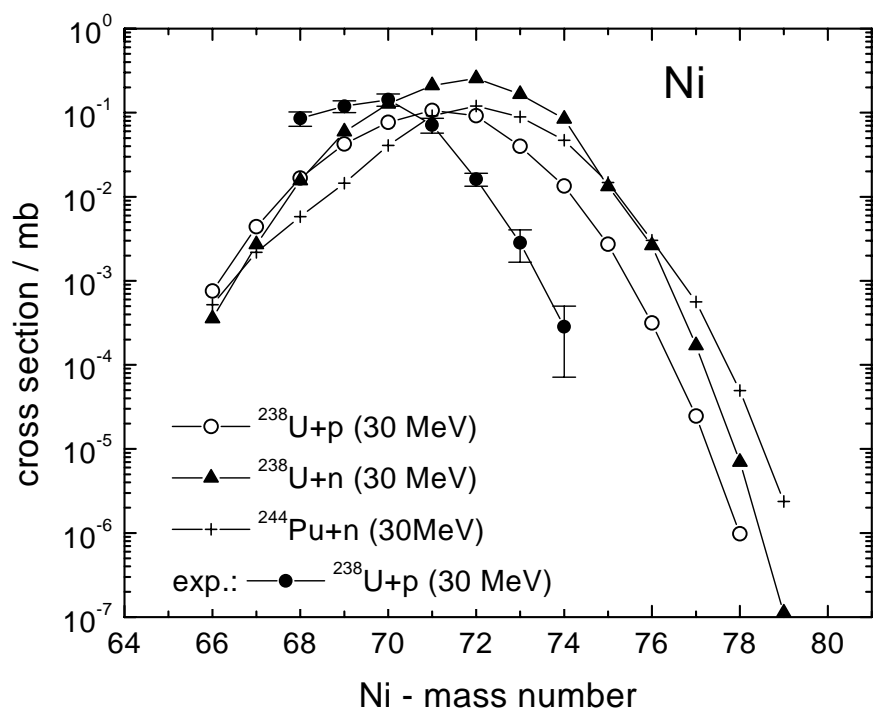

Fig. 2. Comparison of calculated isotopic distributions of $\mathrm{Ni}$ products in proton induced fission of ${ }^{238} \mathrm{U}$ and neutron induced fission of ${ }^{238} \mathrm{U}$ and ${ }^{244} \mathrm{Pu}$ at $30 \mathrm{MeV}$ particle energy, and experimental data (points with error bars) from (20)

there are contributions from three components: the tails from the symmetric and the second asymmetric modes and the superasymmetric mode. Enhancement of fission fragment yields in far asymmetric region is also supported by our time-of-flight measurements with HENDES setup in the neutron induced fission of ${ }^{238} \mathrm{U}$ using reaction ${ }^{238} \mathrm{U}(\mathrm{d}, \mathrm{pf})(19)$. In this case fission fragment mass distributions measured in coincidence with protons for definite proton's energy bins what effectively corresponds to some neutron energy bins in accordance with the reaction kinematics. Predictions for the Ni isotope production cross section in three reactions: ${ }^{238} \mathrm{U}(\mathrm{p}, \mathrm{f}),{ }^{238} \mathrm{U}(\mathrm{n}, \mathrm{f}){ }^{244} \mathrm{Pu}(\mathrm{n}, \mathrm{f})$ at $30 \mathrm{MeV}$ particle energy are presented in Fig. 2 together with experimental data from (20). One can see that the production of extremely neutron-rich ${ }^{78} \mathrm{Ni}$ is highest in fission of the most neutron-rich compound nucleus formed in ${ }^{244} \mathrm{Pu}(\mathrm{n}, \mathrm{f})$ reaction. There is a discrepancy between theoretical results and experimental data (20) for ${ }^{238} \mathrm{U}(\mathrm{p}, \mathrm{f})$ reaction and the reason for this discrepancy is still unclear.

\section{Results on production cross sections by fission}

Calculations with the developed model allow us to predict the production cross sections for different type of fission reaction. In Fig. 3 the Ni isotope production cross sections are compared for four fission reaction: ${ }^{235} \mathrm{U}\left(\mathrm{n}_{t h}, \mathrm{f}\right)$, ${ }^{238} \mathrm{U}(\mathrm{p}, \mathrm{f})$ and ${ }^{238} \mathrm{U}(\mathrm{n}, \mathrm{f})$ at $E_{p(n)}=40 \mathrm{MeV}$, and ${ }^{238} \mathrm{U}(\gamma, \mathrm{f})$ at $E_{\gamma}=14 \mathrm{MeV}$. 


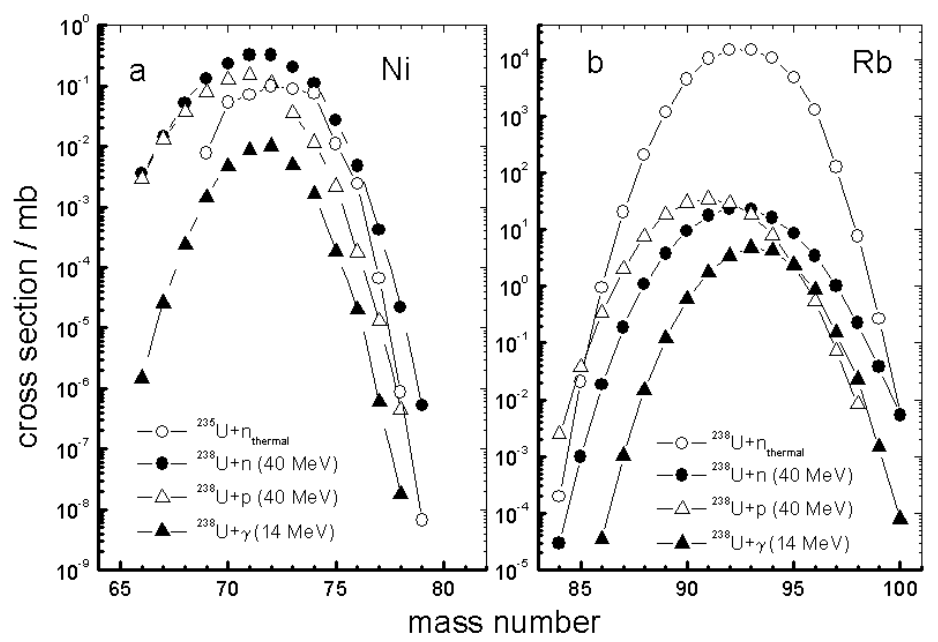

Fig. 3. The $\mathrm{Ni}(\mathbf{a})$ and $\mathrm{Rb}(\mathbf{b})$ isotope production cross sections in reactions: ${ }^{235} \mathrm{U}\left(\mathrm{n}_{\text {th }}, \mathrm{f}\right)$ (open circles), ${ }^{238} \mathrm{U}(\mathrm{n}, \mathrm{f})$ (solid circles), and ${ }^{238} \mathrm{U}(\mathrm{p}, \mathrm{f})$ (triangles) at $E_{p(n)}$ $=40 \mathrm{MeV}$, and ${ }^{238} \mathrm{U}(\gamma, \mathrm{f})$ at $E_{\gamma}=14 \mathrm{MeV}$ (open triangles)

One can note that the production of the very neutron rich $\mathrm{Ni}$ isotopes in the fast neutron induced fission of ${ }^{238} \mathrm{U}$ is higher than in the thermal neutron induced fission of ${ }^{235} \mathrm{U}$. The results of calculations of the cross sections for $\mathrm{Rb}$ isotope production in the same fission reactions are displayed in Fig. 3a. Production of extremly neutron rich nuclei will be higher in fission of more neutron-rich compound nucleus. In this sense, the fast neutron induced fission of ${ }^{238} \mathrm{U}$ should provide a promising method for production and investigation of extremely neutron-rich isotopes.

\section{Conclusion}

In conclusion, a model for calculating the fission product yield in light particle induced fission of heavy nuclei has been developed. This model can be used for the prediction of the formation cross sections of exotic nuclides and for the evaluation of product yields in fission at intermediate energy. Enhancement of highly asymmetric mass and charge division in the proton and neutron fission of ${ }^{238} \mathrm{U}$ in comparison with thermal neutron induced fission is established. Fast neutron induced fission of ${ }^{238} \mathrm{U}$ and ${ }^{244} \mathrm{Pu}$ is efficient reactions for the production of high intensities of neutron-rich isotope beams. 


\section{References}

[1] T.Mehren et al., Phys. Rev. Lett. 77 (1996) 458.

[2] Ch. Engelmann et al, Z. Phys. A352 (1995) 351.

[3] U. Köster et al., in: International Workshop on Research with Fission Fragments, T.v. Edigy et al. (eds), World Scientific, 1997, p.29.

[4] D. Habs, et al., in: International Workshop on Research with Fission Fragments, T.v. Edigy et al. (eds), World Scientific, 1997, p.18.

[5] "Concept for Advanced Exotic Beam Facility Based on ATLAS", Argonne National Laboratory, working "yellow" paper, (February 1995).

[6] M.-G. Saint-Laurent et al., Nouvelles du Ganil 65 (1999) 9.

[7] Yu. Oganessian, Dubna Radioactive Ion Beams, Workshop on Low Energy Radioactive Ion Beams in Dubna, Dubna, Russia, 15-17 Nov. 1999.

[8] C.D. Bowman et al., Nucl. Instr. and Methods A320 (1992) 336.

[9] P.P. Jauho et al, Phys. Rev. C49 (1994) 2036.

[10] V.A. Rubchenya, in: Nuclear Methods for Transmutation of of nuclear Waste, Proc. of International Workshop, Dubna, Russia, 29-31 May 1996, edited by M. Kh. Khankhasayev et al., World Scientific, 1997, p. 110 .

[11] V.A. Rubchenya et al., in: Nuclear Fission and Fission-Product Spectroscopy,: Second International Workshop, edited by G.Fioni et al.,AIP, CP447, 1998, 453.

[12] U. Brosa et a., Phys. Rep. 197 (1990) 167.

[13] B.L. Tracy et al., Phys. Rev. C5 (1972) 222.

[14] V.A. Rubchenya et al., Phys. Rev. C58 (1998) 1587.

[15] D.J Hoffman et al., Phys. Rev. C51 19952597.

[16] I. Tsekhanovich et al., Nucl. Phys. A658 (1999) 217.

[17] M. Huhta et al, Phys. Lett. B405 (1997) 230.

[18] J.L. Sida et al., Nucl. Phys. A502 (1989) 233c.

[19] V.A. Rubchenya et al., in: Fission and Properties of Neutron-Rich Nuclei. Proc. of The Second International Conference, June 28 - July 2, 1999, St Andrews, Scotland, edited by J.H. Hamilton, W.R. Philips, H.K. Carter, World Scientific, 2000, p. 484.

[20] S. Franchoo et al., Phys. Rev. Lett. 81 (1998) 3100. 